\title{
Clustering and rapid long-range motility behavior of bacteria by type IV pili
}

Authors: Chinedu S. Madukoma ${ }^{1}$, Nydia Morales Soto ${ }^{1,2}$, Anne E. Mattingly ${ }^{1}$, Shaun W. Lee $^{2,3,4}$, Joshua D. Shrout ${ }^{1,2,3,4^{*}}$ DNA or extracellular polysaccharides.

*Correspondence to: joshua.shrout@nd.edu

\section{Affiliations:} Dame, Notre Dame, IN, 46556; USA. USA.

2

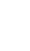

16

\section{Abstract:}

${ }^{1}$ Department of Civil and Environmental Engineering and Earth Sciences, University of Notre

${ }^{2}$ Eck Institute for Global Health, University of Notre Dame, Notre Dame, IN, 46556; USA. ${ }^{3}$ Department of Biological Sciences, University of Notre Dame, Notre Dame, IN, 46556; USA. ${ }^{4}$ Advanced Diagnostics and Therapeutics, University of Notre Dame, Notre Dame, IN, 46556;

Many organisms coordinate to move and colonize over surfaces. Bacteria such as Pseudomonas aeruginosa exhibit such surface motility as a precursor step to forming biofilms. Here we show a group surface motility where small groups of $P$. aeruginosa use their type IV pili (TFP) appendages over long- distances. Small cell clusters employ their TFP to move multiple cell lengths in fractions of a second and form new multicellular groups. Given the length scale and speed of displacement, cells appear to “snap” to a new position and then resume their previous behavior. The same long range TFP action also leads to rapid community contraction of sparsely arranged cell clusters. Cluster development and snapping motility does not require exogenous 


\section{Introduction:}

TFP function as motility appendages by an extension, tip attachment, and retraction order

31 of action [1]. These thin structures ( $6 \mathrm{~nm}$ diameter) range from 0.5-7.0 $\mu \mathrm{m}$ in length and can

32 generate pulling forces exceeding $100 \mathrm{pN}$ [1-5]. For the bacterium Pseudomonas aeruginosa,

33 TFP are known to confer motility phenotypes known as twitching and walking. P. aeruginosa

34 also employs its polar flagellum to confer motility modes called swimming and swarming. The

35 transition between flagellar-mediated motility to TFP-mediated motility is not well understood.

36 For example, while swarming requires active flagella, the absence or over expression of TFP

37 have been shown to influence the overall swarm phenotype [6]. Here we show that TFP facilitate

38 a cluster development and rapid motility phenotype in a subset of cells following flagellar-driven

39 swarming community expansion. We find that $P$. aeruginosa TFP can extend multiple cell

40 lengths $(>30 \mu \mathrm{m})$ and then retract to translocate small clusters of cells to join with other cell

41 clusters in less than one second.

\section{Results and Discussion:}

There are two modes of $P$. aeruginosa surface motility that are routinely investigated

45 using agar motility plate assays. Flagellar-mediated swarming is studied using $0.4 \%-0.6 \%$ agar

46 and TFP-mediated twitching is studied using 1.0\% agar. Both swarming and twitching are

47 commonly assessed in these plate assays as growing and expanding $P$. aeruginosa populations

48 that develop over hours to days. Here we detail surface motility behavior that occurs on surfaces

49 between those conditions typically used to examine swarming and twitching (0.4-1.0\% agar). We

50 find that as P. aeruginosa colonies expand from the point of inoculation, small clusters of cells

51 advance beyond the larger group. Using this approach, an open-air plate was required — covering 
52 the agar (with a glass coverslip) does not promote the same cluster development. We find this

53 clustering phenotype is conserved among $P$. aeruginosa strains, as all common laboratory strains

54 tested (PA14, PAO1, MPAO1, PAK and PAO1C) develop clusters to some degree (Figure S1).

Inspection of these clusters over time shows they are transient and facilitate the

restructuring of the community. Cells cluster, divide into smaller groups, and recluster numerous

“snap” from one region of the frame to another (Movie S1).
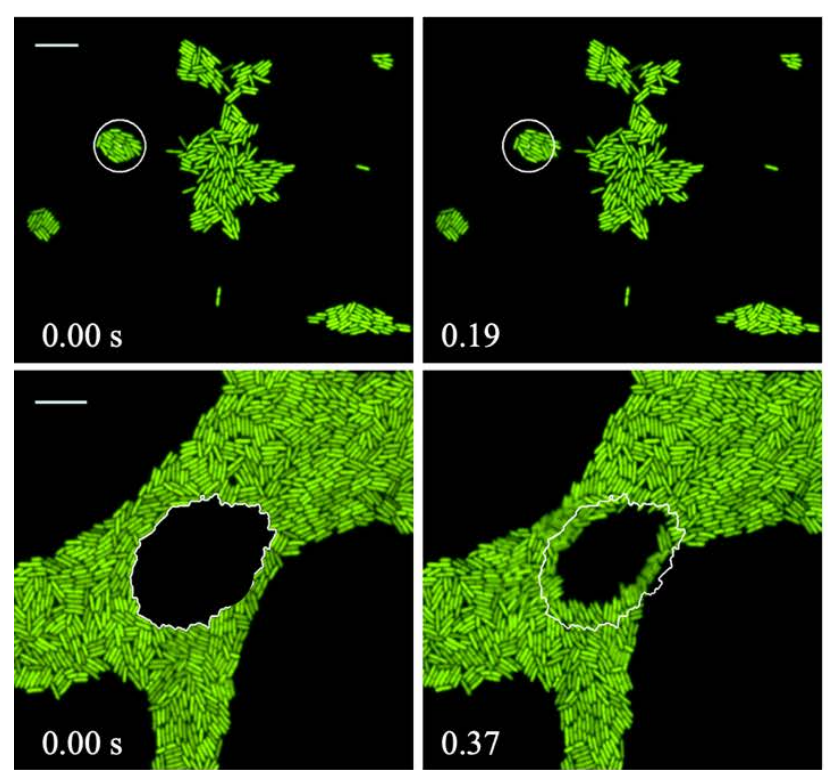
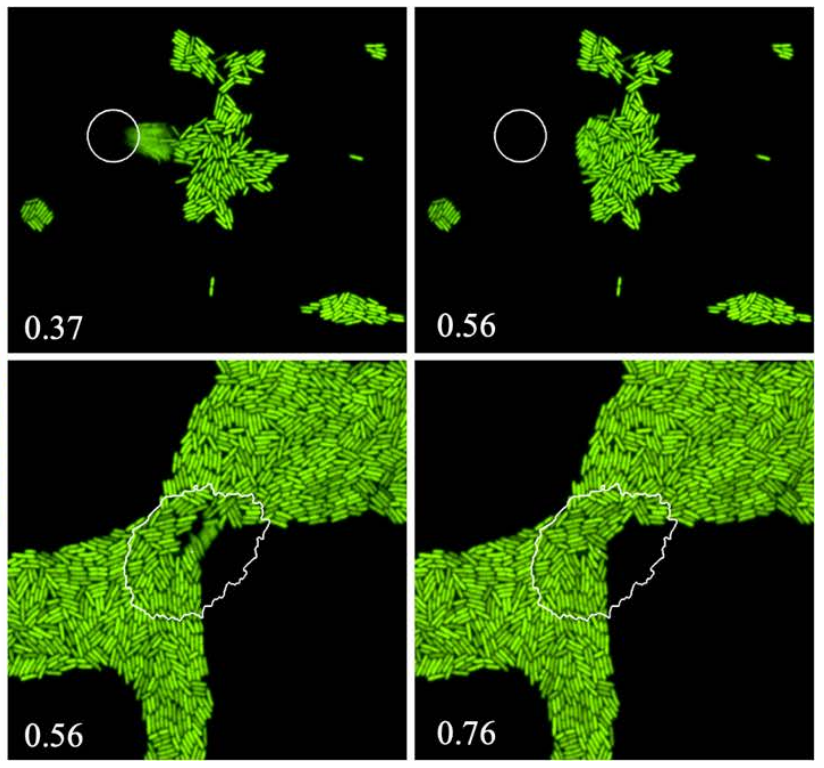

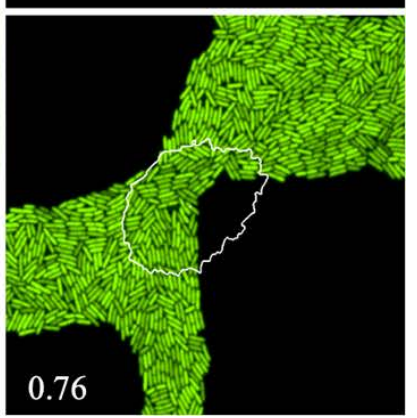

Fig. 1. P. aeruginosa snapping and community contraction in less than 1 second. Time-lapse sequences of representative $P$. aeruginosa snapping and community contraction events are shown. Top row: advancing cluster (identified with white circle at the $0 \mathrm{~s}$ ) prepares to snap by enhacing cluster arrangement (0.19 s), begins moving (0.37 s), and joins another cluster (0.56 s). Bottom row: A contraction snapping event. The open area surrounded by cells (traced in whitespanning $469.87 \mu^{2}$ ) is covered within $0.76 \mathrm{~s}$. Scale bar represents $10 \mu \mathrm{m}$.

In all, we track cells in 115 of these snapping clusters in various assays and specifically quantify 70 snapping events in experiments containing $0.8 \%$ agar (Table 1 ). These snapping clusters contain an average of 17.5 cells and travel a maximum distance of $17.8 \mu \mathrm{m}$, or $\sim 4$ cell lengths. Cluster size does not correlate with distance traveled or snapping duration. Likewise, no 
72 apparent trend changes are attributable to the original time of assay inoculation. However, the

73 total distance traveled by snapping clusters strongly relates with water availability in these

74 surface motility assays. The maximum snapping distance on $0.45 \%$ agar assays is $37 \mu \mathrm{m}$ or $\sim 9$

75 cell lengths, while the maximum distance cells snap on $1.0 \%$ agar is $10 \mu \mathrm{m}$.

76

Table 1. P. aeruginosa snapping behavior dynamic traits.

\begin{tabular}{ll}
\multicolumn{1}{c}{ Trait } & Average $( \pm$ standard deviation) \\
\hline Cells in cluster,** & $17.5 \pm 9.60$ \\
Cluster area at $\mathrm{t}=0\left(\mu \mathrm{m}^{2}\right)$ & $67.2 \pm 39.4$ \\
Cell length & $4.2 \pm 0.75$ \\
Distance travelled $(\mu \mathrm{m})^{*}$ & $11.4 \pm 3.00$ \\
Duration $(\mathrm{sec}) * *$ & $0.478 \pm 0.183$ \\
Cluster velocity $(\mu \mathrm{m} / \mathrm{sec})$ & $26.8 \pm 10.5$
\end{tabular}

78 Quantification from $n=70$ identified snapping events for $P$. aeruginosa growing on $0.8 \%$ agar.

$79 *$ Pearson correlation between cells in cluster and distance travelled is 0.475 .

$80 * *$ Pearson correlation between cells in cluster and duration of event is 0.039 .

83 of community contraction. A representative example is shown in Figure 1 (bottom panels) where

84 an open area surrounded by cells (traced in white—spanning $469.87 \mu^{2}$ ) collapses within 0.76

85 seconds.

86 Snapping behavior requires $P$. aeruginosa TFP. Isogenic mutants deficient for TFP fail to

87 form the small aggregates and no sub-second rapid behavior is detected (Figure 2). Further,

88 functional TFP retraction is required as the retraction-deficient pilU strain also does not form

89 clusters and exhibits behavior like the pilA strain. When one considers the size of PilA

90 monomers [7] and TFP assembly [8] required to mediate snapping, nearly 17,000 PilA

91 monomers are required to assemble TFP capable of the average event measured in our

92 experiments. Accordingly, the pilus required to mediate the maximum distance event (of $37 \mu \mathrm{m}$ )

93 contains a staggering 35,200 PilA monomers. 

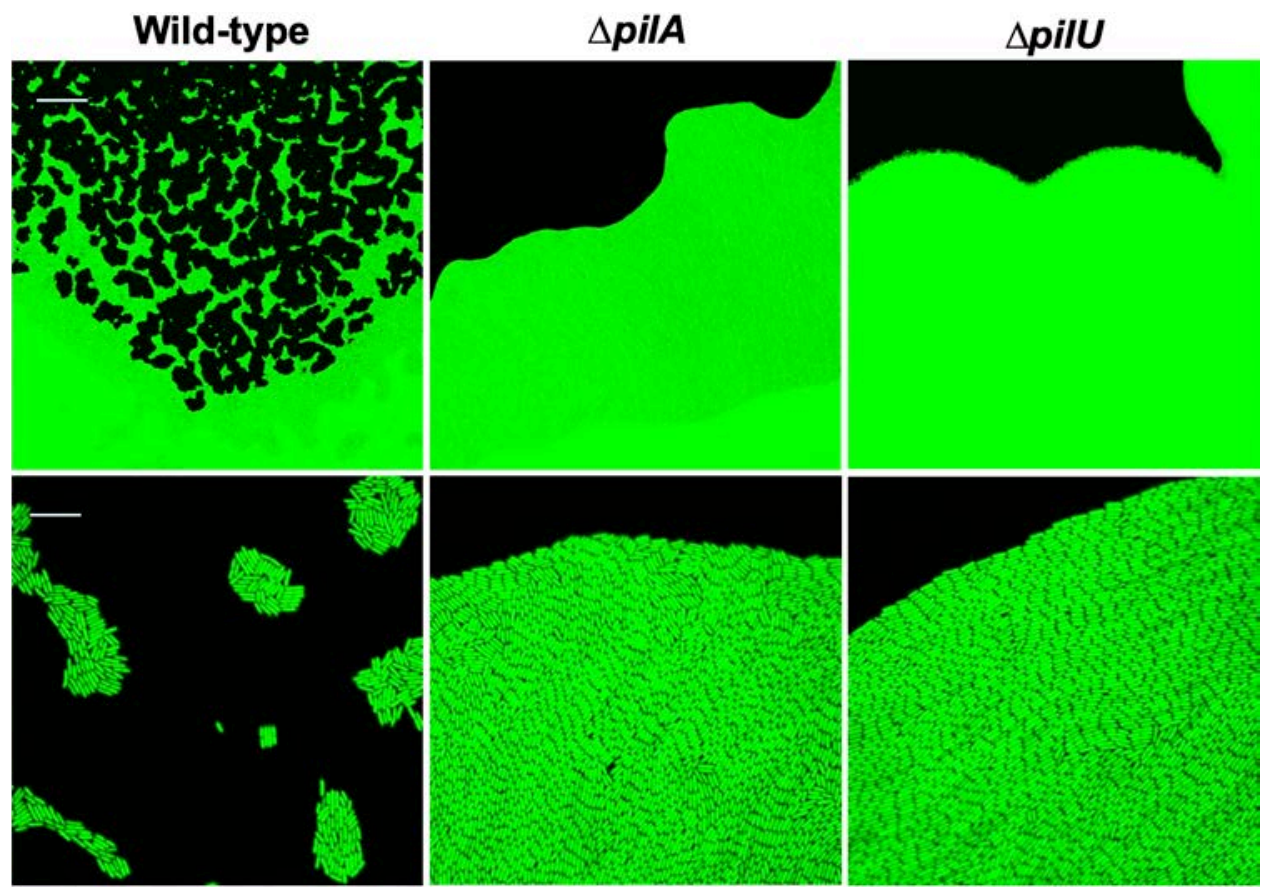

Figure 2. Advancing colony phenotype of $P$. aeruginosa on motility assay plates containing $0.8 \%$ agar. Top (10× magnification) demonstrates cluster development by wild-type beyond the advancing swarm zone. TFP-deficient $(\triangle p i l A)$ and TFP retraction-deficient $(\Delta p i l U)$ strains make no clusters. Bottom row (100× magnification) shows arrangements of single cells, and $\Delta p i l A$ and $\Delta$ pilU appear highly ordered and tightly packed. Scale bars represent $100 \mu \mathrm{m}$ (top) and $10 \mu \mathrm{m}$ (bottom).

A lag time is seen after plate assay inoculation before snapping is observed. We also see

104 that a functional flagellum is a required precursor to this snapping motility phenotype. On $0.5 \%$

105 agar assays that promote robust swarming, the lag time for cluster development and snapping is

106 1.5-2 days (as the plates dry out). However, using an agar range of 0.8\%-1.0\% promotes cluster

107 formation and snapping within a few hours post inoculation. Cells missing their polar flagellum

108 (fliC) are widely dispersed and fail to develop the small clusters that form beyond the advancing

109 large population on these surface assays (Figure 3). Thus, it appears that flagellar-mediated

110 swarming can serve as an initial step to establish the needed population of cells to subsequently

111 form cell clusters that will snap together using their TFP. 
113 additional biological factors required. We find that snapping also requires the surfactant

114 rhamnolipid. Cells from a rhamnolipid-deficient strain ( $\triangle r h l A B)$ can move away from the

115 uniformly expanding $P$. aeruginosa colony and form some clusters, but these bacteria never

116 exhibit snapping motion (Figure 3).
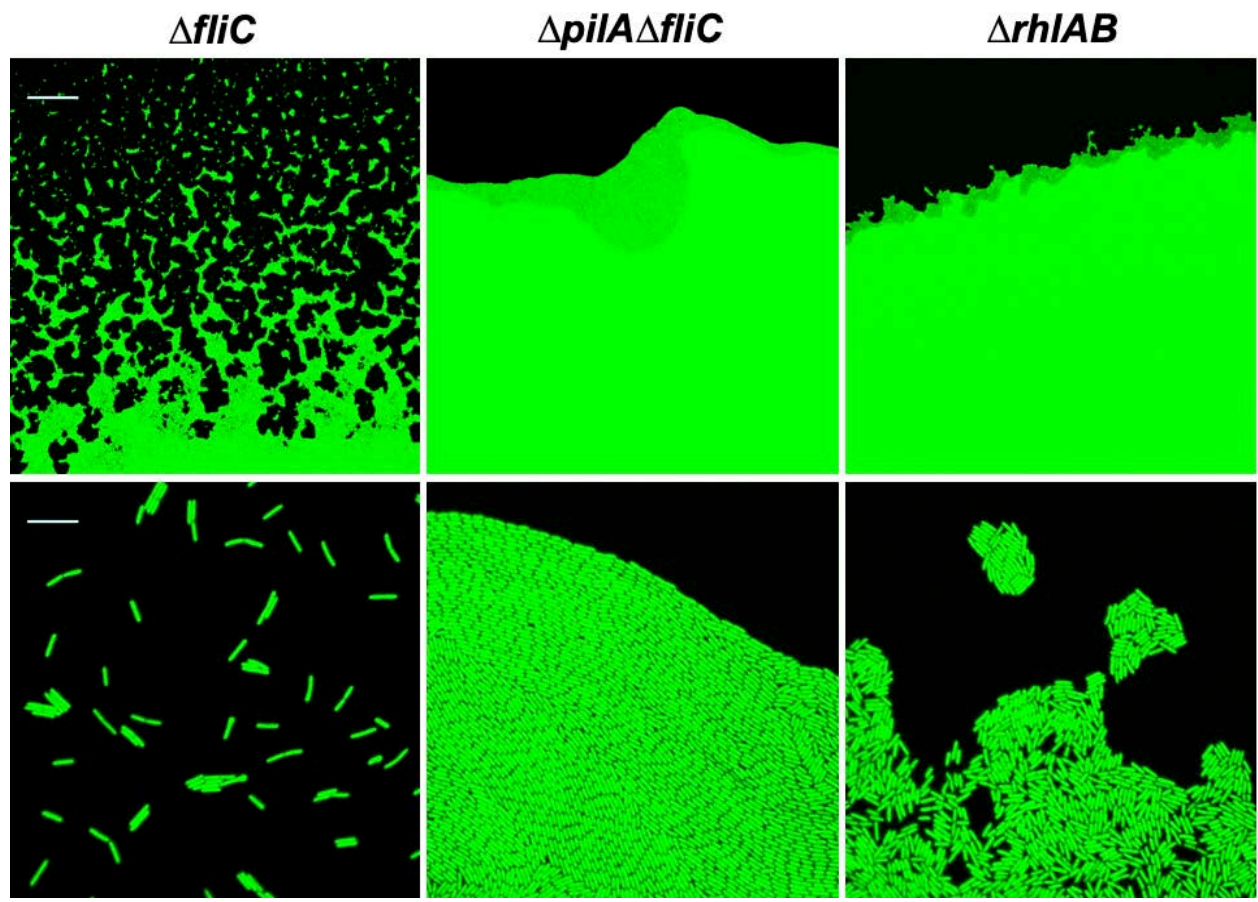

119 Figure 3. Colony edge and clustering phenotype of $P$. aeruginosa mutants on $0.8 \%$ agar. Top 120 (10× magnification): Flagella-deficient $(\Delta$ fliC) are mostly sparsely distributed at the single-cell 121 level, while the appendage-deficient strain $(\triangle p i l A \Delta f l i C)$ do not form cells clusters. Rhamnolipid122 deficient $(\triangle r h l A B)$ cells make a reduced number of smaller clusters closer to the colony edge.

123 Bottom row (100× magnification) show arrangements of single cells (mono-layer). Images were 124 obtained using confocal microscopy twelve hours after inoculation. Top (10× magnification)

We next examine the potential role of DNA as a marker and promoter of snapping 
131 same experiment as "explosive cell lysis” [11]. Figure 4 shows snapping of a small cluster in a

132 region with no exogenous DNA while a cell simultaneously explodes to release DNA in a

133 separate region (sequence included as Movie S2). Thus, we conclude that snapping does not

134 require TFP-DNA interaction and is distinct from TFP actions mediated by exogenous DNA.
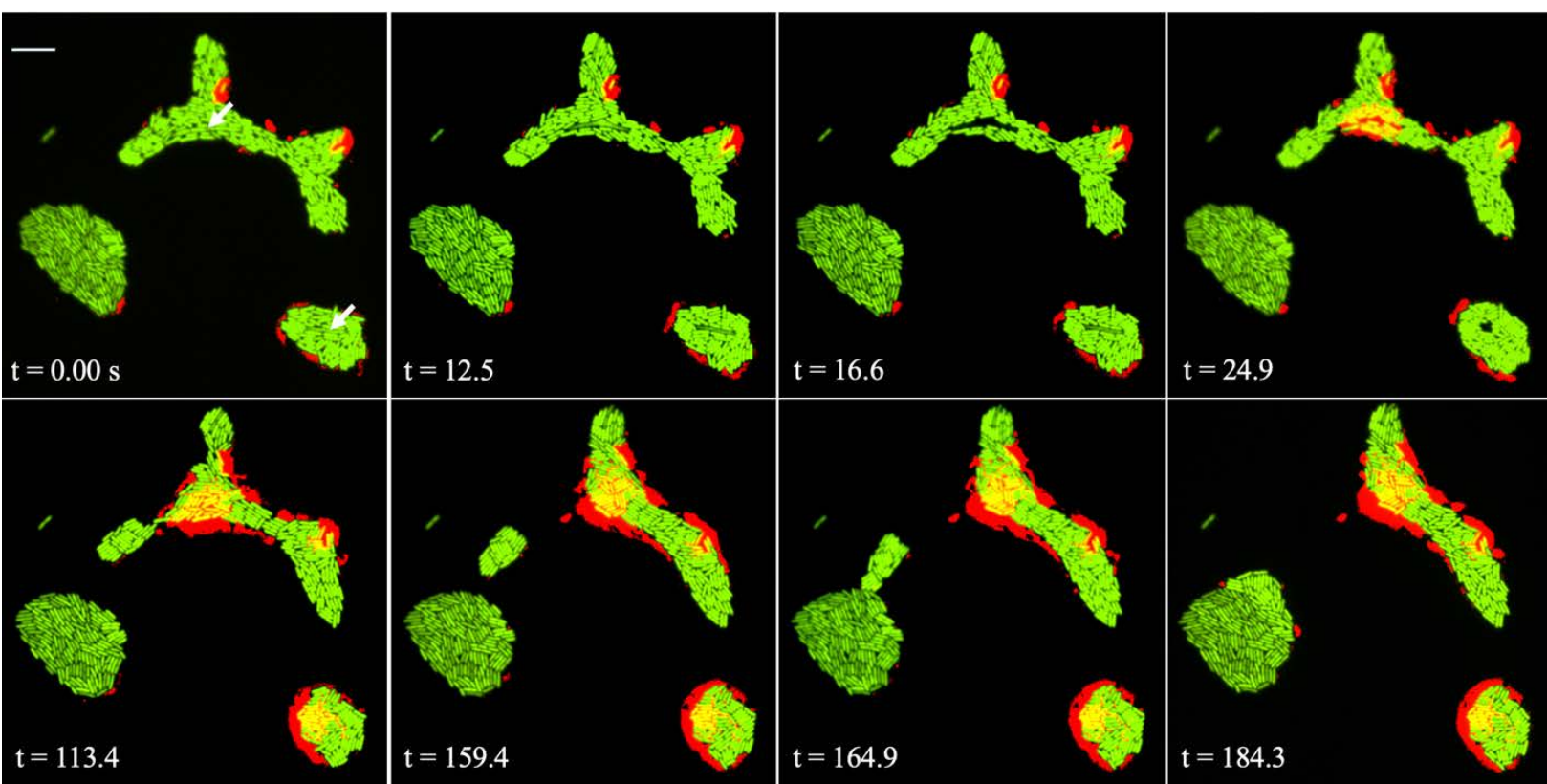
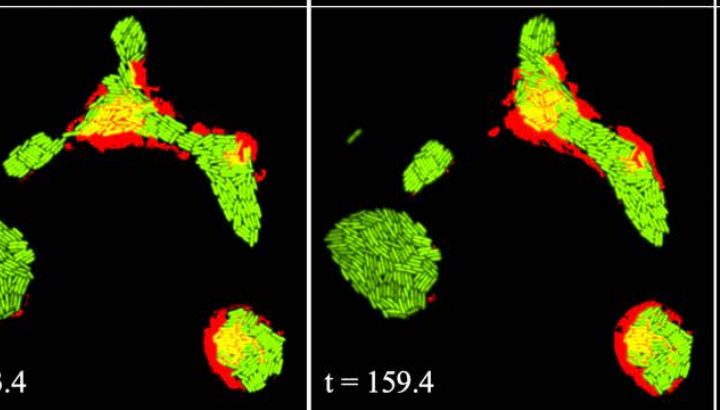

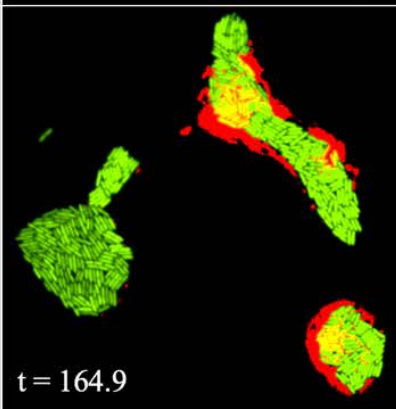

$\mathrm{t}=184.3$

Figure 4. Sequence of events demonstrating eDNA release from a lysing cell within the same time span as snapping motility that occurs elsewhere in the same frame. A cell ready to lyse (top arrow, $0.00 \mathrm{~s}$ ) ceases production of GFP (12.5 s), explodes by $16.6 \mathrm{~s}$, and releases its exogenous DNA at $24.9 \mathrm{~s}$. The red color indicates the DNA released by the lysing cell. A cluster begins to pull away (113.4 s) from the cluster exhibiting a high concentration of high eDNA and snaps onto a different cluster by the $116.9 \mathrm{~s}$. Scale bar represents $10 \mu \mathrm{m}$.

Since DNA did not serve as an attractant for snapping clusters, we next investigate the $P$.

aeruginosa polysaccharides Pel and Psl, which have been shown important to P. aeruginosa surface motility and aggregation as a stage in biofilm development $[12,13]$. Similarly, the bacterium Myxococcus xanthus is well known to require exopolysaccharide to confer "social" TFP-mediated motility [14-16]. However, we find that the Pel and Psl polysaccharides for $P$. aeruginosa do not confer cluster development or the snapping phenotype. Elimination of either 
150 equivalent snapping events to that we observe for the wildtype (Figure 5).

Wild-type
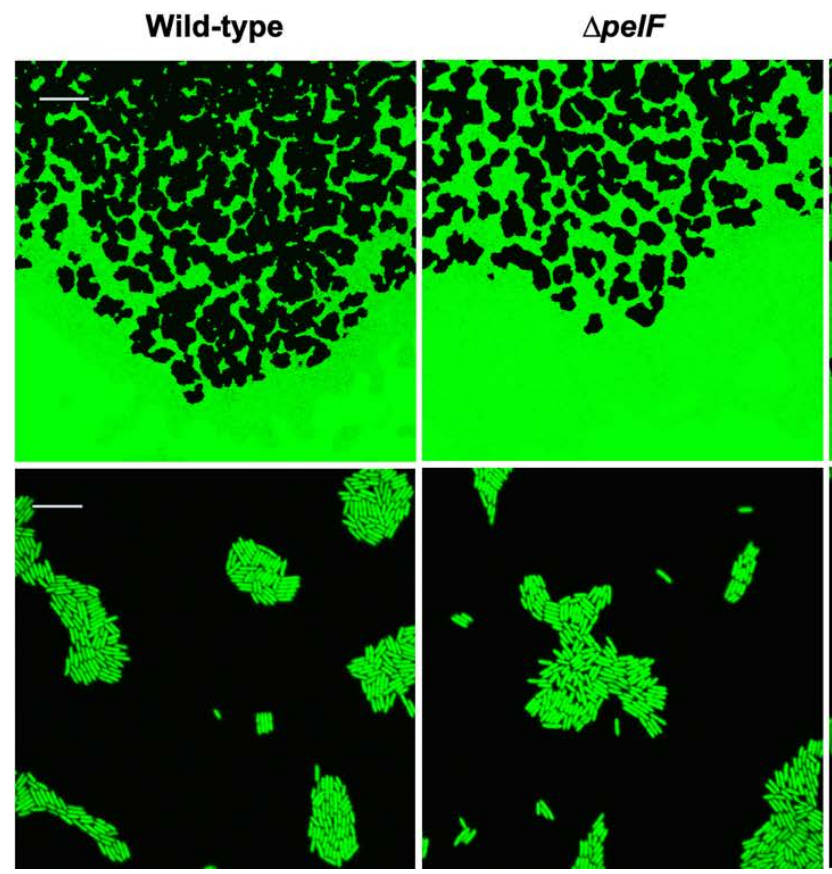

$\triangle p s I A B C$

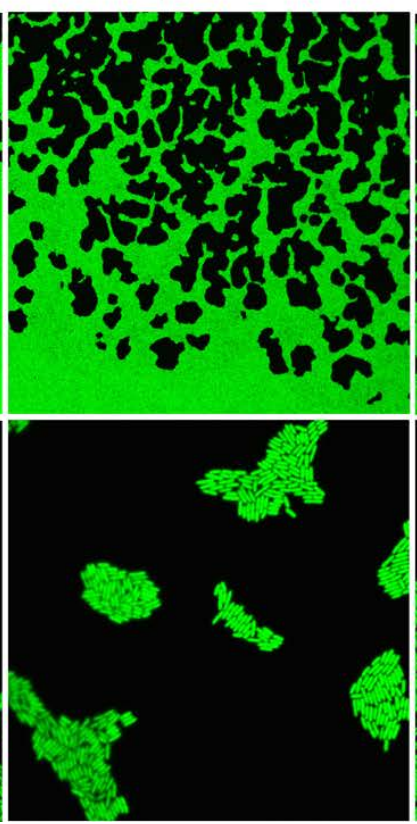

$\triangle p s I A B C \triangle p e I F$

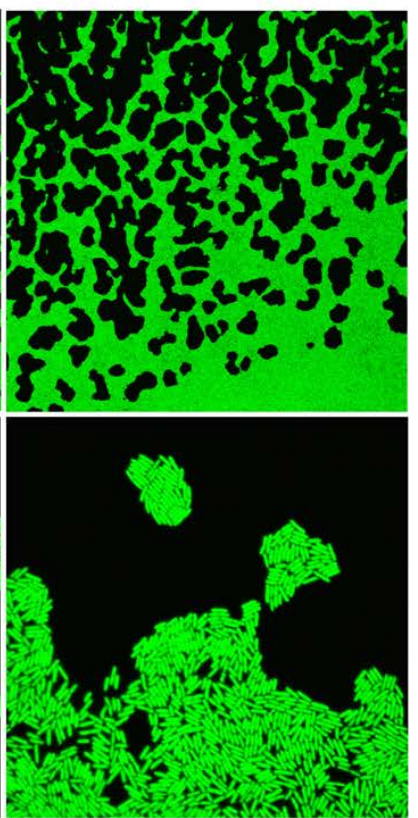

Figure 5. Top (10× magnification) demonstrates cluster formation by Pel-deficient $(\Delta p e l F)$, Psldeficient $(\triangle p s l A B C)$, and polysaccharide-deficient $(\triangle p s l A B C \triangle p e l F)$ in similar fashion as the wildtype. Bottom row (100× magnification) shows the arrangements of single cells (mono-layer) within these clusters and advancing swarm edges. Bottom row (100× magnification) show clusters at of single cell level. Scale bar represent $100 \mu \mathrm{m}$ (top) and $10 \mu \mathrm{m}$ (bottom).

Here we detail TFP-dependent snapping in the bacterium $P$. aeruginosa. Our probing of cluster development on semi-solid surfaces suggests that snapping occurs readily as small groups of $P$. aeruginosa cells rapidly restructure the surrounding community within seconds.

\section{Materials and Methods}

Bacteria strains and growth medium. Strains of Pseudomonas aeruginosa used in this study are included in Table S1. Cultures were streaked from frozen $\left(-80^{\circ} \mathrm{C}\right)$ stocks onto LB agar plates (1.5\% wt/vol) and incubated at $37^{\circ} \mathrm{C}$ overnight. Isolated colonies selected and grown 
167 planktonically in $6 \mathrm{~mL}$ FAB with $30 \mathrm{mM}$ glucose $[6,17]$ at $37^{\circ} \mathrm{C}$ with shaking at $240 \mathrm{rpm}$ for 12 -

16815 hrs.

169 Isogenic mutant construction. Primers and gBLOCK nucleotides used for this work are

170 included in Table S2. Plasmid pCSM1 was constructed to allow creation of a markerless

$171 \triangle$ pslABC mutant. pCSM1 was constructed by inserting a single gBLOCK of double-stranded

172 DNA (IDT, Coralville, IA) containing both regions upstream of the pslA gene and downstream

173 of the pslC gene according to the Pseudomonas genome database [18]; this gBLOCK was

174 restricted using EcoRI and HindIII and ligated into the pEX18Ap vector between the EcoRI and

175 HindIII sites and expressed in E. coli DH5 $\alpha$.

176 Similarly, plasmids pNMS and pCSM103 were constructed to allow creation of a markerless

$177 \Delta r h l A B$ and $\triangle$ fliC mutants. pNMS utilized a single gBLOCK containing both regions upstream

178 of the rhlA gene and downstream of the $r h l B$ gene and pCSM103 contained regions upstream

179 and downstream of the fliC gene. These amplified gBLOCK products were ligated into the

180 pEX18Gm plasmid [19] following SLiCE protocols [20].

181 Mutations were introduced using conjugational mating. Single cross-over recombinants were

182 selected on LB plates (1.5\% agar wt/vol), augmented with $100 \mu \mathrm{g} / \mathrm{ml}$ carbenicillin (pNMS,

183 pCSM1 and pCSM103) or 100 $\mathrm{g} / \mathrm{ml}$ gentamycin $(\mathrm{pEX} 18 \mathrm{Gm} \Delta \mathrm{pelF})$. Double crossover

184 recombinants were then selected on 5\% wt/vol sucrose LB plates. Deletions were confirmed by

185 comparing PCR amplification of the target region with the PAO1C wildtype.

186 Constitutive fluorescent strain construction. Plasmids harboring a Tn7 region containing green

187 fluorescent protein under control of a constitutive strong promoter were incorporated into $P$.

188 aeruginosa strains by conjugational mating. Either plasmid pBK miniTn7-gfp2 (harboring

189 gentamycin resistance) or pBK miniTn7-gfp3 (harboring kanamycin and streptomycin 
resistance) were incorporated adjacent to $\mathrm{g} \operatorname{lmS}$ on the $P$. aeruginosa chromosome using helper

191 plasmid pUX-BF13 and mobilization plasmid pRK600. Transgenic cells were selected on LB

192 agar supplemented with $100 \mu \mathrm{g} / \mathrm{mL}$ gentamycin or $250 \mu \mathrm{g} / \mathrm{mL}$ kanamycin and $250 \mu \mathrm{g} / \mathrm{mL}$

193 streptomycin and confirmed by PCR detection of the chromosomal insertions and microscopy

194 inspection for fluorescence.

195 Surface motility assays. Surface motility plate assays were composed of modified fastidious

196 anaerobe broth (FAB) media that included $0.1 \%$ casamino acid (wt/vol) and no ammonium

197 sulfate $\left(\left(\mathrm{NH}_{4}\right)_{2} \mathrm{SO}_{4}\right)[6,17]$. Surface motility was evaluated over a range of Noble Agar

198 concentrations between $0.4 \%-3.0 \%$ (wt/vol). Plate assays were spot inoculated by pipetting $1 \mu \mathrm{m}$

199 log-phase cells onto the center of the 60mm Petri dish and quickly transferred to a laminar hood

200 for 3-5 minutes with lids partially open, promoting rapid absorption of the inoculation droplet

201 into the motility agar. All plates were inverted and incubated at $30^{\circ} \mathrm{C}$ in a humidity-controlled

202 (85\% RH) incubator for 15-30 h. Prior to imaging, motility plates were equilibrated to room

203 temperature for 45-60 minutes.

204 Microscopy and imaging. Imaging was carried out using a Nikon Eclipse Ti-E Inverted

205 microscope equipped with Plan Fluor 10X DIC LN1 and 100X LU Plan Fluor objectives and

206 Andor DU-888 X-10006 camera with Emission wavelength = 535, Excitation wavelength $=470$.

207 Image processing and analysis. Images were processed using Nikon NIS-Element software and

208 further analyzed using Fiji image processing package (3). Using tools in these software

209 packages, size of clusters, distance and speed travelled by a cluster were determined.

211 References:

2121 Skerker, J.M., and Berg, H.C. (2001). Direct observation of extension and retraction of type IV pili. Proc Natl aeruginosa pili by using atomic force microscopy. J Bacteriol 188, 370-377. 
3 Maier, B., et al. (2002). Single pilus motor forces exceed 100 pN. Proc Natl Acad Sci USA 99, 16012-16017.

4 Merz, A.J., et al. (2000). Pilus retraction powers bacterial twitching motility. Nature 407, 98-102.

5 Clausen, M., et al. (2009). High-force generation is a conserved property of type IV pilus systems. J Bacteriol 191, 4633-4638.

6 Anyan, M.E., et al. (2014). Type IV pili interactions promote intercellular association and moderate swarming of Pseudomonas aeruginosa. Proc Natl Acad Sci USA 111, 18013-18018.

7 Craig, L., et al. (2006). Type IV Pilus Structure by Cryo-Electron Microscopy and Crystallography: Implications for Pilus Assembly and Functions. Molecular Cell 23, 651-662.

8 Chang, Y.-W., et al. (2016). Architecture of the type IVa pilus machine. Science 351.

9 Barken, K.B., et al. (2008). Roles of type IV pili, flagellum-mediated motility and extracellular DNA in the formation of mature multicellular structures in Pseudomonas aeruginosa biofilms. Environ Microbiol 10, 2331-2343.

10 Webb, J.S., et al. (2003). Cell death in Pseudomonas aeruginosa biofilm development. J Bacteriol 185, 45854592.

11 Turnbull, L., et al. (2016). Explosive cell lysis as a mechanism for the biogenesis of bacterial membrane vesicles and biofilms. Nature Communications 7.

12 Sheth, H., et al. (1994). The pili of Pseudomonas aeruginosa strains PAK and PAO bind specifically to the carbohydrate sequence $\beta$ GalNAc (1-4) $\beta$ Gal found in glycosphingolipids asialo-GM1 and asialo-GM2. Mol Microbiol 11, 715-723.

13 Zhao, K., et al. (2013). Psl trails guide exploration and microcolony formation in Pseudomonas aeruginosa biofilms. Nature 497, 388-391.

14 Kaiser, D. (1979). Social gliding is correlated with the presence of pili in Myxococcus xanthus. Proceedings of the National Academy of Science U S A 76, 5952-5956.

15 Youderian, P., and Hartzell, P.L. (2007). Triple mutants uncover three new genes required for social motility in Myхососсиs xanthus. Genetics 177, 557-566.

$16 \mathrm{Lu}$, A., et al. (2005). Exopolysaccharide biosynthesis genes required for social motility in Myxococcus xanthus. Mol Microbiol 55, 206-220.

17 Morales-Soto, N., et al. (2015). Preparation, imaging, and quantification of bacterial surface motility assays. J Vis Exp.

18 Winsor, G.L., et al. (2016). Enhanced annotations and features for comparing thousands of Pseudomonas genomes in the Pseudomonas genome database. Nucleic Acids Res 44, D646-653.

19 Hoang, T.T., et al. (1998). A broad-host-range Flp-FRT recombination system for site-specific excision of chromosomally-located DNA sequences: application for isolation of unmarked Pseudomonas aeruginosa mutants. Gene 212, 77-86.

20 Zhang, Y.W., et al. (2012). SLiCE: a novel bacterial cell extract-based DNA cloning method. Nucleic Acids Res 40.

21 Shrout, J.D., et al. (2006). The impact of quorum sensing and swarming motility on Pseudomonas aeruginosa biofilm formation is nutritionally conditional. Mol Microbiol 62, 1264-1277.

22 Morales-Soto, N., et al. (2018). Spatially dependent alkyl quinolone signaling responses to antibiotics in Pseudomonas aeruginosa swarms. J Biol Chem 293, 9544-9552.

23 Colvin, K.M., et al. (2012). The Pel and Psl polysaccharides provide Pseudomonas aeruginosa structural redundancy within the biofilm matrix. Environ Microbiol 14, 1913-1928.

24 Kessler, B., et al. (1992). A general system to integrate lacZ fusions into the chromosomes of gram-negative eubacteria: regulation of the $P m$ promoter of the TOL plasmid studied with all controlling elements in monocopy. Mol Gen Genet 233, 293-301.

25 Bao, Y., et al. (1991). An improved Tn7-based system for the single-copy insertion of cloned genes into chromosomes of gram-negative bacteria. Gene 109, 167-168.

26 Koch, B., et al. (2001). A panel of Tn7-based vectors for insertion of the gfp marker gene or for delivery of cloned DNA into Gram-negative bacteria at a neutral chromosomal site. J Microbiol Meth 45, 187-195.

Acknowledgements:

268 (SWL). 
bioRxiv preprint doi: https://doi.org/10.1101/525790; this version posted January 21, 2019. The copyright holder for this preprint (which was not certified by peer review) is the author/funder. All rights reserved. No reuse allowed without permission. University of Washington-Seattle. 
Supplementary Materials:

274

275

276

277

278

279
Tables S1-S2

Figure S1

Movies S1-S2

\section{Table S1. Strain list}

\begin{tabular}{|c|c|c|}
\hline Strain or plasmid & Relevant Characteristics & $\begin{array}{l}\text { Source or } \\
\text { reference }\end{array}$ \\
\hline \multicolumn{3}{|l|}{ P. aeruginosa } \\
\hline Wild type (wt) & wildtype PAO1C & {$[6,21]$} \\
\hline wt-GFP & PAO1C::miniTn7 gfp2; $\mathrm{Cm}^{\mathrm{r}}, \mathrm{Gm}^{\mathrm{r}}$ & {$[6,21]$} \\
\hline wt-mCherry & PAO1C::mini-Tn7-Gm-P A1/04/03-mCherry & {$[6]$} \\
\hline$\Delta p i l A$ & PAO1C $\Delta$ pilA; markerless & {$[6,21]$} \\
\hline$\triangle p i l A-G F P$ & $\Delta p i l A$ miniTn7 gfp2; $\mathrm{Cm}^{\mathrm{r}}, \mathrm{Ap}^{\mathrm{r}}, \mathrm{Gm}^{\mathrm{r}}$ & [6] \\
\hline$\Delta p i l U$ & PAO1C $\Delta$ pilU; markerless & [6] \\
\hline$\Delta p i l U-G F P$ & $\Delta p i l U:: \operatorname{miniTn} 7$ gfp2; $\mathrm{Cm}^{\mathrm{r}}, \mathrm{Ap}^{\mathrm{r}}, \mathrm{Gm}^{\mathrm{r}}$ & [6] \\
\hline$\triangle r h l A B$ & PAO1C $\triangle r h l A B$; markerless & This study \\
\hline$\triangle r h l A B-G F P$ & $\Delta r h l A B::$ miniTn7-gfp2; $\mathrm{Cm}^{\mathrm{r}}, \mathrm{Ap}^{\mathrm{r}}, \mathrm{Gm}^{\mathrm{r}}$ & This study \\
\hline$\Delta$ fliC & PAO1C $\Delta$ fliC; markerless & This study \\
\hline$\Delta$ fliC-GFP & PAO1C $\Delta$ fliC::miniTn7-gfp3; $\mathrm{Tc}^{\mathrm{r}}, \mathrm{Km}^{\mathrm{r}}, \mathrm{Sm}^{\mathrm{r}}$ & This study \\
\hline$\Delta p s l$ & PAO1C $\triangle p s l A B C$; markerless & This study \\
\hline$\Delta p s l-G F P$ & $\Delta p s l A B C:: \operatorname{miniTn} 7-g f p 2 ; \mathrm{Cm}^{\mathrm{r}}, \mathrm{Ap}^{\mathrm{r}}, \mathrm{Gm}^{\mathrm{r}}$ & This study \\
\hline$\Delta p e l F$ & PAO1C $\Delta p e l F$; markerless & This study \\
\hline$\Delta p e l F-G F P$ & $\Delta p e l F:: \operatorname{miniTn} 7-g f p 2 ; \mathrm{Cm}^{\mathrm{r}}, \mathrm{Ap}^{\mathrm{r}}, \mathrm{Gm}^{\mathrm{r}}$ & This study \\
\hline$\Delta p s l \Delta p e l$ & PAO1C $\triangle p s I A B C \Delta p e l F$; markerless & This study \\
\hline$\Delta p s l \Delta p e l-G F P$ & $\Delta p s l \Delta p e l:: \operatorname{miniTn} 7-g f p 2 ; \mathrm{Cm}^{\mathrm{r}}, \mathrm{Ap}^{\mathrm{r}}, \mathrm{Gm}^{\mathrm{r}}$ & This study \\
\hline PA14-GFP & wildtype PA14::miniTn7 gfp2; $\mathrm{Cm}^{\mathrm{r}}, \mathrm{Gm}^{\mathrm{r}}$ & {$[22]$} \\
\hline PA14pelF & PA14 $\Delta p e l F$ & {$[23]$} \\
\hline PAO1-GFP & wildtype PA01::miniTn7 gfp2; $\mathrm{Cm}^{\mathrm{r}}, \mathrm{Gm}^{\mathrm{r}}$ & This study \\
\hline MPAO1-GFP & wildtype MPA01::miniTn7 gfp2; $\mathrm{Cm}^{\mathrm{r}}, \mathrm{Gm}^{\mathrm{r}}$ & This study \\
\hline PAK-GFP & wildtype PAK::miniTn7 gfp2; $\mathrm{Cm}^{\mathrm{r}}$, Gm ${ }^{\mathrm{r}}$ & This study \\
\hline \multicolumn{3}{|l|}{$\underline{\text { Plasmids }}$} \\
\hline$\overline{\mathrm{pEX} 18 \mathrm{Ap}}$ & Suicide gene replacement vector, $A p^{r}$ & [19] \\
\hline pRK600 & Mobilization plasmid, $\mathrm{Cm}^{\mathrm{r}}$ & {$[24]$} \\
\hline pUX-BF13 & Helper plasmid, $\mathrm{Ap}^{\mathrm{r}}$ & {$[25]$} \\
\hline pBK-miniTn7-gfp2 & mini Tn7-gfp2; $\mathrm{Cm}^{\mathrm{r}}, \mathrm{Ap}^{\mathrm{r}}, \mathrm{Gm}^{\mathrm{r}}$ & {$[26]$} \\
\hline pBK-miniTn7-gfp3 & mini Tn7-gfp3:: $\mathrm{Cm}^{\mathrm{r}}, \mathrm{Ap}^{\mathrm{r}}, \mathrm{Km}^{\mathrm{r}}, \mathrm{Sm}^{\mathrm{r}}$ & {$[26]$} \\
\hline pNMS & rhlAB allelic replacement vector in pEX18Gm; $\mathrm{Gm}^{\mathrm{r}}$ & This study \\
\hline $\mathrm{pEX} 18 \mathrm{Gm} \Delta p e l F$ & pEX18Gm:: $\Delta$ pelF allelic replacement vector; $\mathrm{Gm}^{\mathrm{r}}$ & {$[23]$} \\
\hline pCSM1 & pslABCD allelic replacement vector in pEX18Ap; $\mathrm{Amp}^{\mathrm{r}}$ & This study \\
\hline pCSM103 & fliC allelic replacement vector in pEX18Gm; $\mathrm{Gm}^{\mathrm{r}}$ & This study \\
\hline
\end{tabular}




\section{Table S2. Primers and gBLOCK sequences}

\begin{tabular}{ll}
\hline Primers & Sequence $\left(5^{\prime} \rightarrow 3^{\prime}\right)$ \\
\hline PelF-up & ACCAGCAGGATGCGTTTGTA \\
rhlAB-fown & TGGTACTGGGAACTGGCCTA \\
rhlAB-R & CACGCTGAGCAAATTGTTCG \\
rhlAB gBLOCK & CGAAGATCTACGCCAATGAAGG \\
& GCTCTGGTCGAACAGGCTGTCGCTCCAGACCACCATTTCCGAGGAGCGCAGGCCGT \\
& TGAGGATCGCCGGATCCACGGCCCCGTAGTTCTGCATCTGGTATCGCTCCAGCCAG \\
& GCCTTGGGATAGGTGCCATGGACCTCGGTCTTCGGCCGGGTGAAGGGAATCGTGTG \\
& GCGCACGCCATAGGCGTAGTAATCGAAGCCCAGGCGCCGCACTTCCTTTCCAGGA \\
& CGGCGAACACGCCCTGGCTGTCGTGGATCGGCTGCATCTCGCTACGCAAACCGTCC \\
& CACCACAGCAAAAAGCCTCCGTCATTCCTCATTGCAGTAAGCCCTGATCGATAAAA \\
& TGCATCACAGCAGAATTGGCCCGGGGTATGACGCTGTCAAGCGCGCAGCCTTGCCG \\
& ATACGGCAAAATCATGGCAACCCTATCTGTTATGCCAGCACCGTTCAGGACCGCAT \\
& TTCACACCTCCCAAAAATTTCGAACAGGCAAACAGCTATCGCTGCCACGGGTATC \\
& CCGGCATTACGTAGAGTTCGTTCTTATTGTTCGAACGGCAGACAAGTAACTCAGCG \\
& GCCATCCGCGCGGACCAGCCACGGCGGATGCGTACCCCTTCAGGCGAGGGGCTTGT \\
& GTGGGTCTTGCAGATCGGCCTGCGCAAACGATTGGCGTCCGTGTTCACGCGTAGCC \\
& GATGAACACTTTTAGCCAATTCGAAAGCTAACGGTAACTGCCAGATTCACAGGA \\
& CGGGCGGCCGCCTGCGCATAAGCCCCTGCCCGCCCGCGATAAGGCGTGCCAGAGC \\
& GGCCGGCGGGGAACAGCGAGGGGTTGGCGGAAAAGGCATGAAAAAACCGGGCGC \\
& CAGGCCCGGTTTCCGGTGTCGAAACGCTCTGGCGTCAGGCTTTTGGCAGGGTCACG \\
& CCGCGCTGGCCCTGGTATTAAGCTTGGCACTGGCCGTCGTTTTACAACGTC
\end{tabular}

fliC-F

fliC-R

fliC GBlock
TGCTTTGCTATCGCGACAGTC

\section{GGCCATAATCCATCTCCGTCAAA}

TGCTTTGCTATCGCGACAGTCTCCCGGTCACGCGGGTGAAGAACCGTTCCAGGGACT GGCTGGAGGCGATCCGCGCGCACCTGGCCGATGCAGACGCCAACGCCGCGGCCGGC GCGCAGTTGCGCGAGGCGGTGCGCCGCGACTGGATGCTCGAAGGCGCGCATCTCGA GGCATGGGCGGCAGCCTGGCTGCCGGACTGAATCCGGGTTTTTCTCGAACGAGGCC GGTGGCGCAAGCCATCGGCCTTTTTTCATGCCCGGCGTGCCCTGTTGCACGGGAGGG CTAAAGAAAATCGCCGGGGGGTCGATGCAATGGGTGTCGGAACTTCCACCCTCTGC CGGACCAACCGGGGGCGGTTCAGGACCGATATTGGCGAGTCCTCTTCGAAGCATGT AACCCACTGAAGAGGAAGAGAAAAAGAAAATGTTGATTTTTTCTCTAAAGCTCCGC CGGGAAACGCCGATAAACACCATGAACGCGAATTCTTGGGGCACCTGAGCAAGCAG GCCGAGAGATCGCAAGCTCAGGTAACCGAAATAGGTCCTTTGGAGGAAATCACCAT GGCCCGCTAAGCCCGGGAACGGTCACTCACGCGGTACTGGGAGGAAGGGGTGACCC TTCСTCССТTTTCССTTTGCGAGGCATGAGAAATGGACGTCGGAAATATCACTTCCC TTTCTACGTTCAAGACCGGCAGGGCCCCGGAGGCCAGCGGCGATATCTTTGCGCCGC GCAGCGCAGCGGATGGCAGCGGCAAACCGTTGCCGGAAGTGACGGCTTCCCGGGAG GCCAGCGAATCTCGCGATGACCTGGGGCTCGCCGTCAGCGACATCCAGTCTTTCGTG CAGAGCGTCAAGCGCAACTTGAACTTCAGCATCGACGACTCCTCTGGCCAGGTCGT GGTCAAGGTGATCGACGGTGACTCGGGCGAAGTCGTGCGGCAAATCCCTTCCGAAG AAGTACTAAAACTTGCCGCCCGGCTCGACGACGTACACAGTGTCCTGTTCGAAACCC GCGCCTGACGCGCGGGTTGAACGACTTGGCATGGTGCTTGCCCTATCGAAGGGATA GGGCAGTACATGCACGGTTTTTTGACGGAGATGGATTATGGCC 
bioRxiv preprint doi: https://doi.org/10.1101/525790; this version posted January 21, 2019. The copyright holder for this preprint (which was not certified by peer review) is the author/funder. All rights reserved. No reuse allowed without permission.

\begin{tabular}{ll} 
pslABC-F & TACGGCCAGTACCTGAACTACCA \\
pslABC-R & TTCCGGCCCGATCTTCATCAA \\
\hline pslABC & GCTTGAATTCGGCAAACGTCCGCCAAGCCCCCCAGGTCGGACCGGCACGCTCGAAC \\
& GGCCAACCTGCGTCATGGAACCGCAGGCGCATCCTGCCCAGCCAGCCCGCAGCGCC \\
& AGTGGACAGGCGGGTCGTCGAACCGGCGGCTGGCCACCTGGCCGAGCGGCCTGCCC \\
& TCACCTTTCGCCCCGCTTCGCTTCCCAGGCCAGAGCGCTCGCGGATTGGCGGCGTCA \\
& GATTTCCTCGTCTACTGTTTGGATAAAAGTTTGGCGCCAGAAATACGTCAATAAATT \\
& GACTAAAAAAACTTACCCAGACTACGGATATTTCCCTGGGAATGCTAAGATAGCTA \\
& TCACAAAGCCACTATCGACGAATGAACCTATTCGACGGGAAAATGACTAAACGCG \\
& TGGCAAATGAAAAATAGTCACTAAATTGACGCTTCACCGCCTTGCTCTTCCCTATCC \\
& ACTCAATGGACTGCCCGTGATCGGCAGAGCAAACAACACATCATGACCTACAGGAA \\
& GTGCTCCCTCATGAAACGCACCCTCCTCATGCTCGCCATGCTCGCCCTGGCCGCATG \\
& CAACACCCCCGCACGGATTCCCGCACCGGACAGCGACACCGTGGACAGCGGCAAGC \\
& GTGCCCTGGAAGAACTCGCCAGGCTACCGCCGGCGATGGAGCGGGTGCGCGTCGGG \\
& GACACCCTGCGGATCGTCCGCGATGCCGGGGAGATGCCGACCCTCTCGGCGTTCAA \\
& CGTCGCCACCATCTATGAACTGACGCTGTACACCGTGCTCAACGACGGCAGCATCTA \\
& CTATCCGTTCATCGGTCGCATCCAGGCCGCGCACCGCACGCCGCAGGAAATCGCCA \\
& ACGAGCTGACCACCAAGCTCGCGCCGATCTACCGCGAGCCGCGGGTCACGGTGAAC \\
& ATCAACCAGGCGCCGGGCAATACGGTGTTCGTCGGCGGCGCGGTGCGCAACCCGTC \\
GGCCGTGCCGATCCCCGCCGCCAACAACATGAAGCTTGCTT
\end{tabular}




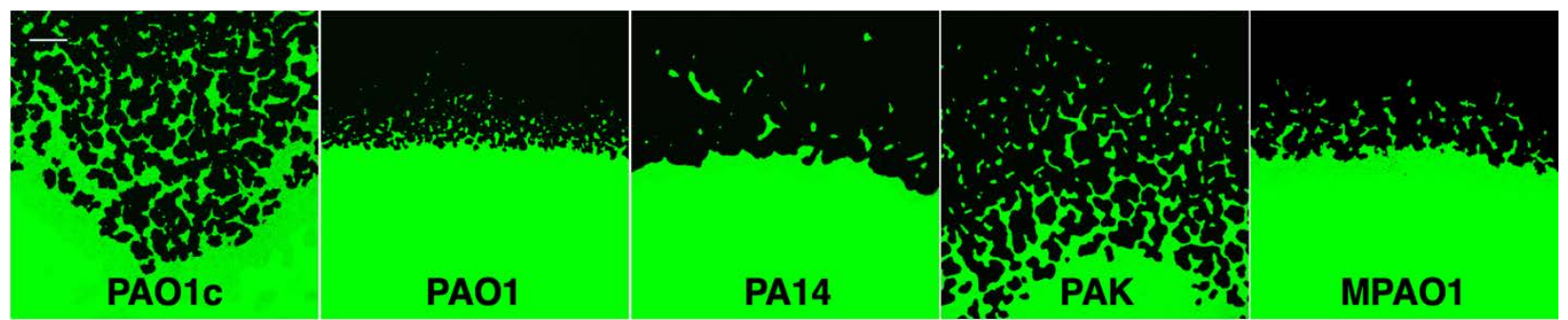

284 Fig. S1. Cluster formation is conserved among wild-type P. aeruginosa strains on $0.8 \%$ agar. Scale

\section{Supplemental Movie Captions:}

Movie S1. P. aeruginosa snapping motility. The cluster in the upper right of frame undergoes some cell-cell rearrangement and then snaps to the larger cluster from right-to-left. (5.3 frames per second)

Movie S2. P. aeruginosa rapid community contraction. The open area surrounded by cells 